\title{
CLASSIFICATION OF SECANT DEFECTIVE MANIFOLDS NEAR THE EXTREMAL CASE
}

\author{
KANGJIN HAN
}

(Communicated by Lev Borisov)

\begin{abstract}
Let $X \subset \mathbb{P}^{N}$ be a nondegenerate irreducible closed subvariety of dimension $n$ over the field of complex numbers and let $S X \subset \mathbb{P}^{N}$ be its secant variety. $X \subset \mathbb{P}^{N}$ is called 'secant defective' if $\operatorname{dim}(S X)$ is strictly less than the expected dimension $2 n+1$. In a 1993 paper, F.L. Zak showed that for a secant defective manifold it is necessary that $N \leq\left(\begin{array}{c}n+2 \\ n\end{array}\right)-1$ and that the Veronese variety $v_{2}\left(\mathbb{P}^{n}\right)$ is the only boundary case. Recently R. Muñoz, J. C. Sierra, and L. E. Solá Conde classified secant defective varieties next to this extremal case.

In this paper, we will consider secant defective manifolds $X \subset \mathbb{P}^{N}$ of dimension $n$ with $N=\left(\begin{array}{c}n+2 \\ n\end{array}\right)-1-\epsilon$ for $\epsilon \geq 0$. First, we will prove that $X$ is an $L Q E L$-manifold of type $\delta=1$ for $\epsilon \leq n-2$ by showing that the tangential behavior of $X$ is good enough to apply the Scorza lemma. Then we will completely describe the above manifolds by using the classification of conicconnected manifolds given by Ionescu and Russo. Our method generalizes previous results by Zak, and by Muñoz, Sierra, and Solá Conde.
\end{abstract}

\section{INTRODUCTION}

Let $X \subset \mathbb{P}^{N}$ be a nondegenerate irreducible closed subvariety of dimension $n$ over the field of complex numbers. If $X$ is also smooth, we call it a manifold. Let $S X \subset \mathbb{P}^{N}$ be the secant variety of $X$, i.e. the closure of the union of all lines which pass through two or more points of $X$.

Clearly, we know that $\operatorname{dim}(S X)$ is less than or equal to $\min \{N, 2 n+1\}$. If $\operatorname{dim}(S X)<2 n+1$, then $X \subset \mathbb{P}^{N}$ is said to be secant defective and we call $\delta(X):=$ $2 n+1-\operatorname{dim}(S X)>0$ the secant defect of $X$.

It is well-known that there are no secant defective curves if $N \geq 3$. For surfaces, in Sev01] F. Severi proved that the second Veronese embedding $v_{2}\left(\mathbb{P}^{2}\right)$ in $\mathbb{P}^{5}$ and cones are the only secant defective surfaces in $\mathbb{P}^{N}, N \geq 5$. Later G. Scorza classified all secant defective irreducible varieties of dimension 3 in $S \mathrm{Sc0} 08$, a result rediscovered by T. Fujita in the smooth case in Fuj82 (see also CC01 for a modern revisitation of Scorza's original paper $[\mathrm{Sco08}])$. In dimension 4, some partial results have been known (e.g., see [Sco09] and FR81]), but a complete classification in arbitrary dimension seems to be out of reach until now.

Received by the editors August 31, 2011 and, in revised form, January 27, 2012 and February 23,2012 .

2010 Mathematics Subject Classification. Primary 14Mxx, 14Nxx, 14M22.

Key words and phrases. Secant defective, local quadratic entry locus, conic-connected, Terracini lemma, tangential projection, second fundamental form, Scorza lemma.

This work was supported by the National Research Foundation of Korea (NRF) with a grant funded by the Korean government (MEST) (No. 2011-0001182).

(C) 2013 American Mathematical Society Reverts to public domain 28 years from publication 
One could expect that the secant variety $S X$ becomes larger as $N$ increases. In Zak93. Zak proved an upper bound for the embedding dimension of secant defective manifolds:

Theorem 0.1 (Zak). Let $X \subset \mathbb{P}^{N}$ be a nondegenerate manifold of dimension $n \geq 2$ with $\operatorname{dim}(S X) \leq 2 n$ and let $M(n):=\left(\begin{array}{c}n+2 \\ 2\end{array}\right)-1$. Then, $N \leq M(n)$ with equality holding if and only if $X$ is the second Veronese embedding $v_{2}\left(\mathbb{P}^{n}\right) \subset \mathbb{P}^{M(n)}$.

Therefore, for secant defective manifolds $X \subset \mathbb{P}^{N}$ it is meaningful to investigate manifolds whose embedding dimension is near this upper bound for $N$, i.e. $M(n)$. Let $B^{n} \subset \mathbb{P}^{M(n)-1}$ be the projection of $v_{2}\left(\mathbb{P}^{n}\right) \subset \mathbb{P}^{M(n)}$ from a point on it. Using tangential projections, R. Muñoz, J. C. Sierra, and L. E. Solá Conde gave a first answer in [MSS07] as follows:

Theorem 0.2 (Muñoz, Sierra, Solá Conde). Let $X \subset \mathbb{P}^{N}$ be a nondegenerate secant defective manifold of dimension $n$ and let $N \geq \max \{M(n)-1,2 n+1\}$. Then one of the following conditions holds:

(a) $n \geq 2$ and $X$ is the second Veronese embedding $v_{2}\left(\mathbb{P}^{n}\right) \subset \mathbb{P}^{M(n)}$;

(b) either $n \geq 3$ and $X$ is the isomorphic projection of $v_{2}\left(\mathbb{P}^{n}\right) \subset \mathbb{P}^{M(n)-1}$ or $n \geq 3$ and $X$ is $B^{n} \subset \mathbb{P}^{M(n)-1}$.

Here we will consider the secant defective manifolds $X \subset \mathbb{P}^{N}$ of dimension $n$ with $N=M(n)-\epsilon$ for some $\epsilon \geq 0$. We will first show that $X$ is an $L Q E L$-mainfold for relatively small $\epsilon \geq 0$ (see Theorem 2.1) by investigating a general tangential projection via the second fundamental form and deducing that the general tangential behavior of $X$ is good enough to apply the Scorza lemma. Next, we give a classification of secant defective manifolds for the cases $\epsilon \leq n-2$ (see Corollary 2.3) using the classification of conic-connected manifolds in [R10] due to P. Ionescu and F. Russo.

\section{Preliminaries}

We work over the field of complex numbers. Let $X \subset \mathbb{P}^{N}$ be a nondegenerate irreducible closed submanifold of dimension $n$.

Let $x, y \in X$ be two general points and $p$ be a general point on the line $\langle x, y\rangle$. We call the closure of the locus of couples of distinct points of $X$ spanning secant lines passing through $p$ as the entry locus of $X$ with respect to $p \in S X$ and denote it by $\Sigma_{p}(X)$. For a general $p \in S X$, it is easy to see that $\Sigma_{p}(X)$ is equidimensional of dimension $\delta(X)$. We would like to recall that for two given general points $x, y \in X$ there exists an $r$-dimensional quadric hypersurface $Q_{x, y}^{r} \subseteq X$ for some $r \geq 0$ which passes through $x, y$. Clearly, $Q_{x, y}^{r} \subseteq \Sigma_{p}(X)$ when $p \in\langle x, y\rangle$ and $r \leq \delta$. Manifolds for which $r \geq 1$ are called conic-connected manifolds (abbr. CCM), and at the other extreme case (i.e. $r=\delta$ ) they are called manifolds with local quadratic entry locus (abbr. LQELM). Moreover, when $Q_{x, y}^{r}=\Sigma_{p}(X)$ we call $X$ a manifold with quadratic entry locus (abbr. QELM); see [Rus08, IR10].

There is also a classical tool, called the Terracini lemma, which is essential to understanding secant varieties (see the classical reference [Ter11] and the more recent Ad87]). 
Theorem 1.1 (Terracini lemma). Let $X \subset \mathbb{P}_{K}^{N}$ be an irreducible subvariety. Then:

(1) for every $x, y \in X(x \neq y)$, and for every $p \in\langle x, y\rangle$,

$$
\left\langle T_{x} X, T_{y} X\right\rangle \subseteq T_{p} S X
$$

(2) if $\operatorname{char}(K)=0$, there exists an open subset $U$ of $S X$ such that

$$
\left\langle T_{x} X, T_{y} X\right\rangle=T_{p} S X
$$

for every $p \in U$ and $x, y \in X(x \neq y)$ such that $p \in\langle x, y\rangle$.

Tangential projections have been basic tools in the study of secant defective manifolds and of the invariant $\delta(X)$. The tangential projection of $X \subset \mathbb{P}^{N}$, say $\pi_{x}$, is the projection of $X$ from a general (projective) tangent space to $X$ at a general point $x \in X$, indicated by $T_{x} X$. Let $W_{x} \subset \mathbb{P}^{N-n-1}$ be the Zariski closure of the image of $\pi_{x}$ and let $\delta=\delta(X)$. Then, we have

$$
\pi_{x}: X \rightarrow W_{x} \subset \mathbb{P}^{N-n-1},
$$

where $\operatorname{dim}\left(W_{x}\right)=n-\delta$ by the Terracini lemma (Theorem 1.1). In particular the general fiber of $\pi_{x}$ is purely $\delta$-dimensional.

When $X \subset \mathbb{P}^{N}$ is secant-defective and $S X \subsetneq \mathbb{P}^{N}$, we can describe the image of the general tangential projection, $W_{x} \subset \mathbb{P}^{N-n-1}$, via the second fundamental form of $X$ at $x$. Let $\phi: B l_{x} X \rightarrow X$ be the blow-up of $X$ at $x, E=\mathbb{P}\left(\left(T_{x} X\right)^{*}\right)=$ $\mathbb{P}^{n-1} \subset B l_{x} X$ be the exceptional divisor, and $H$ be a hyperplane section of $X \subset \mathbb{P}^{N}$. Consider the rational map $\tilde{\pi}_{x}: B l_{x} X \rightarrow \mathbb{P}^{N-n-1}$ and its restriction to $E$ which is given by the linear system $\left|\phi^{*}(H)-2 E\right|_{\mid E} \subseteq\left|-2 E_{\mid E}\right|=\left|\mathcal{O}_{\mathbb{P}^{n-1}}(2)\right|$. We call this linear system the second fundamental form of $X \subset \mathbb{P}^{N}$ at $x$ and denote it by $\left|I I_{x, X}\right|$. Obviously, $\operatorname{dim}\left(\left|I I_{x, X}\right|\right) \leq N-n-1$ and $\tilde{\pi}_{x}(E) \subseteq W_{x} \subseteq \mathbb{P}^{N-n-1}$.

Let $\mathcal{L}_{x}$ be the Hilbert scheme of lines contained in $X$ and passing through $x \in$ $X$. The scheme $\mathcal{L}_{x}$ can be naturally identified with a subscheme of $E \simeq \mathbb{P}^{n-1}$ parametrizing the space of tangent diriections to $X$ at $x$. Then

$$
\left.\mathcal{L}_{x} \subseteq B s\left(\left|I I_{x, X}\right|\right) \text { (the base locus of }\left|I I_{x, X}\right|\right),
$$

since the base locus of $\left|I I_{x, X}\right|$ consists of directions associated to lines having a contact at least three with $X$ at $x$.

Here we recall a key result which allows us to see the whole general tangential projection $W_{x}$ via the second fundamental form $\left|I I_{x, X}\right|$ (see [R09], Proposition 1.2).

Proposition 1.2. Let $X$ be a nondegenerate secant defective manifold and let $x$ be a general point of $X$. Then $\operatorname{dim}\left(\left|I I_{x, X}\right|\right)=N-n-1$ and $\left|I I_{x, X}\right|$ is surjective (i.e. $\left.\tilde{\pi}_{x}(E)=W_{x}\right)$.

It is also remarkable that good tangential behavior guarantees simple entry loci of secant defective manifolds, as the following classical result of Scorza assures (see, for example, [Sco08, Rus08]):

Theorem 1.3 (Scorza lemma). Let $X \subset \mathbb{P}^{N}$ be an irreducible nondegenerate $n$ dimensional variety of secant defect $\delta(X)=\delta \geq 1$ such that $S X \subsetneq \mathbb{P}^{N}$. Suppose that a general tangential projection $\pi_{x}(X)=W_{x} \subset \mathbb{P}^{N-n-1}$ is an irreducible variety having a birational Gauss map. Let $y \in X$ be a general point. Then:

(a) The irreducible component of the closure of fiber of the rational map $\pi_{x}$ : $X \rightarrow W_{x} \subset \mathbb{P}^{N-n-1}$ passing through $y$ is either an irreducible quadric hypersurface of dimension $\delta$ or a linear space of dimension $\delta$, the last case occurring only for singular varieties. 
(b) There exists on $X \subset \mathbb{P}^{N}$ a $2(n-\delta)$-dimensional family $\mathcal{Q}$ of quadric hypersurfaces of dimension $\delta$ such that through two general points $x, y \in X$ there passes a unique quadric $Q_{x, y}$ of the familiy $\mathcal{Q}$. Furthermore, the quadric $Q_{x, y}$ is smooth at $x, y$ and consists of the irreducible components of $\Sigma_{p}(X)$ passing through $x$ and $y, p \in\langle x, y\rangle$ in general.

(c) If $X$ is smooth, then a general member of $\mathcal{Q}$ is smooth.

In particular, we deduce from part (b) of Theorem 1.3 that as soon as $W_{x}$ has a birational Gauss map, then $X \subset \mathbb{P}^{N}$ is a LQELM of type $\delta=\delta(X)>0$ and in particular a $C C M$.

Remark 1.4. Note that it is enough to assume that a general tangential projection $W_{x}$ has a generically finite Gauss map in order to deduce that $X$ is a LQELM via the Scorza lemma. In fact, in the case of characteristic zero, generic finiteness of the Gauss map $\mathcal{G}_{W_{x}}$ is equivalent to the fact that $\mathcal{G}_{W_{x}}$ is birational by linearity of a general contact loci (see [Zak93, Theorem 2.3 (c), pg. 21) and Zak's Theorem on Tangencies (see [Zak93], Corollary 1.8, pg. 18).

Recently, a classification of $C C M$ has been obtained by Ionescu and Russo in [IR10]. Recall that $X$ is Fano if $-K_{X}$ is ample. We call $X$ a prime Fano of index $i(X)$ if $\operatorname{Pic}(X) \simeq \mathbb{Z}\left\langle\mathcal{O}_{X}(1)\right\rangle$ and $-K_{X}=i(X) H$ for some positive integer $i(X)$, where $H$ is the hyperplane section class of $X \subset \mathbb{P}^{N}$. Since conic-connectedness is stable under isomorphic projection, we may assume that $X \subset \mathbb{P}^{N}$ is linearly normal.

Theorem 1.5 (Ionescu, Russo). Let $X \subset \mathbb{P}^{N}$ be a linearly normal CCM of dimension $n$. Then either $X \subset \mathbb{P}^{N}$ is a prime Fano manifold of index $i(X) \geq \frac{n+1}{2}$ or it is projectively equivalent to one of the following:

(a) the second Veronese embedding $v_{2}\left(\mathbb{P}^{n}\right)$ in $\mathbb{P}^{M(n)}$;

(b) the projection of $v_{2}\left(\mathbb{P}^{n}\right)$ from the linear space $\left\langle v_{2}\left(\mathbb{P}^{s}\right)\right\rangle$, where $\mathbb{P}^{s} \subset \mathbb{P}^{n}$ is a linear subspace such that $N=M(n)-\left(\begin{array}{c}s+2 \\ 2\end{array}\right)$ and $0 \leq s \leq n-2$, equivalently $X \simeq B l_{\mathbb{P}^{s}}\left(\mathbb{P}^{n}\right)$ embedded in $\mathbb{P}^{N}$ by the linear system of quadric hypersurfaces of $\mathbb{P}^{n}$ passing through $\mathbb{P}^{s}$;

(c) the Segre embedding $\mathbb{P}^{a} \times \mathbb{P}^{b} \subset \mathbb{P}^{a b+a+b}$, where $a, b \geq 1$ and $a+b=n$;

(d) a hyperplane section of the Segre embedding $\mathbb{P}^{a} \times \mathbb{P}^{b} \subset \mathbb{P}^{N+1}$, where $n \geq 3$, $N=a b+a+b-1, a, b \geq 2$ and $a+b=n+1$.

Remark 1.6. We would like to mention that every prime Fano $X$ of index $i(X) \geq$ $\frac{n+1}{2}$ in the classification of conic-connected manifolds of Theorem 1.5 is actually covered by lines; i.e., through each point of $X$ there passes a line contained in $X$. When $i(X)>\frac{n+1}{2}$, this comes from S. Mori's work in Mor79. In the case of $i(X)=\frac{n+1}{2}$, it is one of the consequences of CMSB02 due to K. Cho, Y. Miyaoka and N.I. Shepherd-Barron.

\section{Secant Defective manifolds near the extremal Case}

Here are our main results.

Theorem 2.1. Let $X \subset \mathbb{P}^{N}$ be a nondegenerate secant defective manifold of $d i$ mension $n \geq 2$ with $S X \subsetneq \mathbb{P}^{N}$ and let $N=M(n)-\epsilon$ for some $\epsilon \geq 0$. Then:

(i) if $\epsilon \leq n-2$, then $\delta(X)=1$, while for $n-1 \leq \epsilon$, we have

$$
1 \leq \delta(X) \leq \min \left\{\epsilon-n+2, \frac{n}{2}\right\}
$$


(ii) if $\epsilon \leq n-2$, then $X \subset \mathbb{P}^{N}$ is a LQELM of type $\delta(X)=1$ (in particular, $X$ is a $C C M)$.

Proof. Let $\delta=\delta(X)>0$. Zak's Linear Normality Theorem (see, for example, Zak93, Corollary 2.17, pg. 48) assures that under our hypothesis, $\delta \leq \frac{n}{2}$. Take a general point $x \in X$ and consider the tangential projection $\pi_{x}: X \subset \mathbb{P}^{N} \rightarrow W_{x} \subset$ $\mathbb{P}^{N-n-1}$. From Proposition 1.2 , we have a diagram

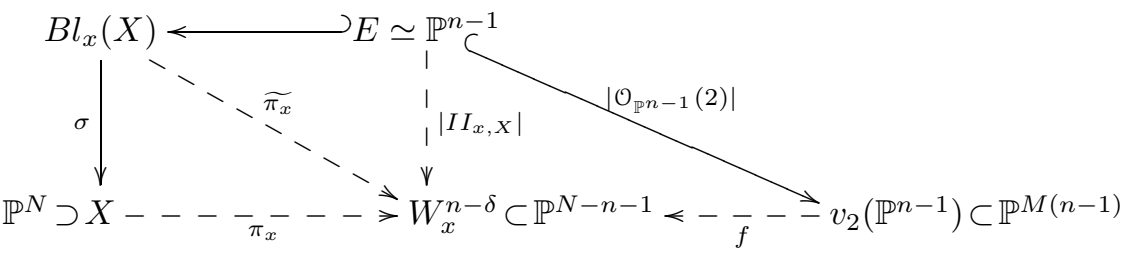

Then

$$
\begin{aligned}
\operatorname{dim}\left|\mathcal{O}_{\mathbb{P}^{n-1}}(2)\right|-\operatorname{dim}\left|I I_{x, X}\right| & =M(n-1)-\{M(n)-\epsilon-n-1\} \\
& =\frac{(n-1)(n+2)}{2}-\frac{n(n+3)}{2}+\epsilon+n+1=\epsilon,
\end{aligned}
$$

so that $f$ (see the diagram (2.1)) should be a projection of the second Veronese $Y:=v_{2}\left(\mathbb{P}^{n-1}\right)$ from a linear subspace $\Lambda \simeq \mathbb{P}^{\epsilon-1}$. Moreover $\delta(Y)=1$, and for any two distinct points $y_{1}, y_{2} \in Y$ we have $\operatorname{dim}\left\langle T_{y_{1}} Y, T_{y_{2}} Y\right\rangle=2 n-2$. Let $y_{1}, y_{2} \in Y$ be two distinct points such that $f\left(y_{1}\right)$ and $f\left(y_{2}\right)$ are smooth points of $W_{x}$ and such that

$$
T_{f\left(y_{1}\right)} W_{x}=T_{f\left(y_{2}\right)} W_{x} \text { and }\left\langle\Lambda, T_{y_{i}} Y\right\rangle=\left\langle\Lambda, T_{f\left(y_{i}\right)} W_{x}\right\rangle \text { for each } i=1,2 .
$$

Then $\left\langle T_{y_{1}} Y, T_{y_{2}} Y\right\rangle \subseteq\left\langle\Lambda, T_{f\left(y_{i}\right)} W_{x}\right\rangle$, yielding

$$
2 n-2 \leq \operatorname{dim}\left\langle\Lambda, T_{f\left(y_{i}\right)} W_{x}\right\rangle=(n-\delta)+\epsilon \leq n-1+\epsilon,
$$

which implies $\delta \leq \epsilon-n+2$ and $\epsilon \geq n-1$.

(i) If $\epsilon \leq n-2$, then by the above argument $f$ is birational and $\delta(X)=1$. Suppose now that $\epsilon \geq n-1$. If $f$ is birational, then $1=\delta(X) \leq \min \left\{\epsilon-n+2, \frac{n}{2}\right\}$; if $f$ is not birational, then by the above argument $\delta(X) \leq \epsilon-n+2$ and part (i) is completely proved.

(ii) Let $y^{\prime}$ be a general point of $W_{x} \subset \mathbb{P}^{N-n-1}$ and suppose that $\mathcal{G}_{W_{x}}$ is not birational. Then, by linearity of general contact loci and Zak's Theorem on Tangencies (see Remark 1.4), we know that there exists an (affine) line $L \subset W_{x}$ passing through $y^{\prime}$ along which the tangent spaces to $W_{x}$ are constant.

By applying the above argument to points $y_{1}, y_{2} \in Y$ lying in two distinct fibers over general points of $L$, we would deduce $\epsilon \geq n-1$, contrary to the hypothesis in (ii). Therefore, part (ii) follows by applying the Scorza lemma (see Theorem 1.3 and Remark 1.4).

Remark 2.2. Here are some remarks on Theorem 2.1

(a) In Zak93 Zak proved that for a secant defective manifold $X \subset \mathbb{P}^{N}$ of dimension $n$ with $\delta=\delta(X)$, necessarily $N \leq g\left(\left[\frac{n}{\delta}\right], n, \delta\right)$; see Zak93] for the definition of the function $g$. Then one easily sees that $g\left(\left[\frac{n}{2}\right], n, 2\right)<$ $M(n)-n+2$, so that the first part of (i) in the above theorem is also a consequence of more general results, which could also provide a proof of (ii) 
above. The above proof is elementary and simple and uses slightly different techniques.

(b) It is obvious that one cannot expect that any secant defective manifold $X \subset$ $\mathbb{P}^{M(n)-\epsilon}$ is a CCM for all $\epsilon \geq 0$. Let us consider an example of some infinite family of 4-dimensional secant defective manifolds, which is well-known (see also FR81]). Let $V=\mathbb{P}^{2} \times \mathbb{P}^{2}$ be a Segre 4-fold in $\mathbb{P}^{8}$ which is a secant defective manifold with $\delta(V)=2$. Choose a point $p \in \mathbb{P}^{9} \backslash \mathbb{P}^{8}$ and consider the cone $Z=C_{p}(V) \subset \mathbb{P}^{9}$. Since $S Z=C_{p}(S V)$, the secant variety $S Z$ is 8-dimensional. Take the intersection of $Z$ and $H$, a general hypersurface of degree $d \geq 2$ not passing through $p$. Denote this 4-dimensional intersection by $X$. Then, $X \subset \mathbb{P}^{9}$ becomes smooth, $S X=S Z=C_{p}(S V), \operatorname{dim}(S X)=8$ and $K_{X} \sim(d-4) H$. Therefore, for a large $d>0, X$ is of general type.

Finally, we present a classification of $n$-dimensional secant defective manifolds $X \subset \mathbb{P}^{N}$ near the extremal case, that is to say, the cases of $N=M(n)-\epsilon$ for $\epsilon \leq n-2$, which extends the previous results of [Zak93, MSS07].

Corollary 2.3. Let $X \subset \mathbb{P}^{N}$ be a nondegenerate secant defective manifold of dimension $n \geq 2$ with $S X \subsetneq \mathbb{P}^{N}$ and let $N \geq M(n)-(n-2)$. Then $X$ is projectively equivalent to one of the following:

(i) the second Veronese embedding $v_{2}\left(\mathbb{P}^{n}\right)$ in $\mathbb{P}^{M(n)}$;

(ii) the isomorphic projection of $v_{2}\left(\mathbb{P}^{n}\right)$ into $\mathbb{P}^{M(n)-\epsilon}$ for any $1 \leq \epsilon \leq n-2$;

(iii) the projection of $v_{2}\left(\mathbb{P}^{n}\right)$ from the linear space $\left\langle v_{2}\left(\mathbb{P}^{s}\right)\right\rangle$ (we call this $B_{s}^{n}$ ), where $\mathbb{P}^{s} \subset \mathbb{P}^{n}$ is any linear subspace such that $s \geq 0,\left(\begin{array}{c}s+2 \\ 2\end{array}\right) \leq n-2$;

(iv) the isomorphic projection of $B_{s}^{n} \subset \mathbb{P}^{M(n)-\left(\begin{array}{c}s+2 \\ 2\end{array}\right)}$ into $\mathbb{P}^{M(n)-\epsilon}$ for any $\epsilon$ with $\left(\begin{array}{c}s+2 \\ 2\end{array}\right)<\epsilon \leq n-2$.

Proof. By Theorem 2.1 any secant defective manifold $X \subset \mathbb{P}^{N}$ is an LQELM for $N=M(n)-\epsilon$, where $0 \leq \epsilon \leq n-2$ and $n \geq 2$. In particular, $X$ is one in the list of the $C C M$ classification of Theorem 1.5. It is easy to see that the second Veronese $v_{2}\left(\mathbb{P}^{2}\right) \subset \mathbb{P}^{5}$ is the only case for $n=2$. From now on, let us assume that $n \geq 3$.

First, we claim that $X \subset \mathbb{P}^{N}$ is not prime Fano in our range. Recall that $\overline{\mathcal{L}}_{x} \subset E$ is the Hilbert scheme of lines contained in $X$ and passing through the general point $x \in X$, which under this hypothesis is equidimensional. From (1.2) we have $\mathcal{L}_{x} \subseteq$ $B s\left(\left|I I_{x, X}\right|\right)=B s(f)$ (the base locus). Since $\delta(X)=1$, Theorem 2.3 in Rus09] yields $\operatorname{dim} \mathcal{L}_{x}=\frac{n-3}{2} \geq 0$. Note that the dimension of the linear span of $v_{2}\left(\mathcal{L}_{x}\right)$ is greater than or equal to $\left(\frac{n-3}{2}+2\right)-1$ with equality holding if and only if $\mathcal{L}_{x} \subset E$ is a linear subspace, a case which under the prime Fano hypothesis is excluded by Theorem 1.1 in Ara06. Furthermore, we have $\left\langle v_{2}\left(\mathcal{L}_{x}\right)\right\rangle \subseteq\langle B s(f)\rangle \subseteq \mathbb{P}^{\epsilon-1}$ so that

$$
\left(\begin{array}{c}
\frac{n-3}{2}+2 \\
2
\end{array}\right)-1<\operatorname{dim}\left\langle v_{2}\left(\mathcal{L}_{x}\right)\right\rangle \leq \epsilon-1 \leq n-3
$$

which is impossible.

Since $\delta\left(\mathbb{P}^{a} \times \mathbb{P}^{b}\right)=2$, we have only to exclude case (d) in Theorem 1.5. In this case

$$
M(n)-\epsilon \geq \frac{n(n+3)}{2}-(n-2)>\frac{(n+3)}{2} \frac{(n+3)}{2}-2 \geq(a+1)(b+1)-2,
$$

where $a \geq 2, b \geq 2, a+b=n+1$, and $n \geq 3$.

This completes the proof. 
With the help of Corollary 2.3. for instance, we could classify all secant defective 5 -folds whose embedding dimension is near the upper bound $M(5)=20$ as follows:

Example 2.4 (Secant defective 5-folds near the extremal case). Let $X$ be a secant defective 5 -fold in $\mathbb{P}^{N}$ with $N \geq M(5)-3$ (i.e. $\left.N=17,18,19,20\right)$. Then, $X$ is one of the following:

(i) the second Veronese embedding $v_{2}\left(\mathbb{P}^{5}\right)$ in $\mathbb{P}^{20}$;

(ii) either the isomorphic projection of $v_{2}\left(\mathbb{P}^{5}\right)$ into $\mathbb{P}^{19}$ or $B_{0}^{5}$ in $\mathbb{P}^{19}$;

(iii) either the isomorphic projection of $v_{2}\left(\mathbb{P}^{5}\right)$ into $\mathbb{P}^{18}$ or the isomorphic projection of $B_{0}^{5}$ into $\mathbb{P}^{18}$;

(iv) either the isomorphic projection of $v_{2}\left(\mathbb{P}^{5}\right)$ into $\mathbb{P}^{17}$, the isomorphic projection of $B_{0}^{5}$ into $\mathbb{P}^{17}$ or $B_{1}^{5}$ in $\mathbb{P}^{17}$.

\section{ACKNOWLEDGEMENTS}

The author would like to thank Professor Paltin Ionescu for having introduced him to this problem during PRAGMATIC 2010, for his encouragement and for valuable suggestions. He is also grateful to the organizers of PRAGMATIC 2010 for providing a good chance to participate and to Dr. José C. Sierra for his useful comments on this work. Finally, he thanks the referee for many corrections and helpful suggestions making this paper more readable.

\section{REFERENCES}

[Ad87] Bjørn Ådlandsvik, Joins and higher secant varieties, Math. Scand. 61 (1987), no. 2, 213-222. MR947474 (89j:14030)

[Ara06] Carolina Araujo, Rational curves of minimal degree and characterizations of projective spaces, Math. Ann. 335 (2006), no. 4, 937-951, DOI 10.1007/s00208-006-0775-2. MR2232023 (2007d:14037)

[CC01] L. Chiantini and C. Ciliberto, Threefolds with degenerate secant variety: on a theorem of G. Scorza, Geometric and combinatorial aspects of commutative algebra (Messina, 1999), Lecture Notes in Pure and Appl. Math., vol. 217, Dekker, New York, 2001, pp. 111-124. MR1824221(2002d:14088)

[CMSB02] Koji Cho, Yoichi Miyaoka, and N. I. Shepherd-Barron, Characterizations of projective space and applications to complex symplectic manifolds, Higher dimensional birational geometry (Kyoto, 1997), Adv. Stud. Pure Math., vol. 35, Math. Soc. Japan, Tokyo, 2002, pp. 1-88. MR.1929792 (2003m:14080)

[Fuj82] Takao Fujita, Projective threefolds with small secant varieties, Sci. Papers College Gen. Ed. Univ. Tokyo 32 (1982), no. 1, 33-46. MR674447 (84d:14023)

[FR81] Takao Fujita and Joel Roberts, Varieties with small secant varieties: the extremal case, Amer. J. Math. 103 (1981), no. 5, 953-976, DOI 10.2307/2374254. MR630774 (82k:14042)

[IR09] P. Ionescu and F. Russo, Manifolds covered by lines, defective manifolds and a restricted Hartshorne conjecture, preprint (arXiv:0909.2763).

[IR10] Paltin Ionescu and Francesco Russo, Conic-connected manifolds, J. Reine Angew. Math. 644 (2010), 145-157, DOI 10.1515/CRELLE.2010.054. MR2671777 (2011f:14083)

[Mor79] Shigefumi Mori, Projective manifolds with ample tangent bundles, Ann. of Math. (2) 110 (1979), no. 3, 593-606, DOI 10.2307/1971241. MR554387 (81j:14010)

[MSS07] Roberto Muñoz, José Carlos Sierra, and Luis E. Solá Conde, Tangential projections and secant defective varieties, Bull. Lond. Math. Soc. 39 (2007), no. 6, 949-961, DOI 10.1112/blms/bdm098. MR2392818 (2009b:14098)

[Rus08] F. Russo, Geometry of Special Varieties, preprint.

[Rus09] Francesco Russo, Varieties with quadratic entry locus. I, Math. Ann. 344 (2009), no. 3, 597-617, DOI 10.1007/s00208-008-0318-0. MR2501303 (2010i:14092) 
[Sco08] G. Scorza, Determinazione delle varietà a tre dimensioni di $S_{r}, r \geq 7$, i cui $S_{3}$ tangenti si tagliano due a due, Rend. Circ. Mat. Palermo 25 (1908), 167-182.

[Sco09] G. Scorza, Sulle varietà a quattro dimensioni di $S_{r}, r \geq 9$, i cui $S_{4}$ tangenti si tagliano a due a due, Rend. Circ. Mat. Palermo 27 (1909), 148-178.

[Sev01] F. Severi, Intorno ai punti doppi impropri di una superficie generale dello spazio a quattro dimensioni e ai suoi punti tripli apparenti, Rend. Circ. Mat. Palermo 15 (1901), $33-51$.

[Ter11] A. Terracini, Sulle $V_{k}$ per cui la varietà degli $S_{h}(h+1)$-secanti ha dimensione minore dell' ordinario, Rend. Circ. Mat. Palermo 31 (1911), 392-396.

[Zak93] F. L. Zak, Tangents and secants of algebraic varieties, Translations of Mathematical Monographs, vol. 127, American Mathematical Society, Providence, RI, 1993. Translated from the Russian manuscript by the author. MR.1234494 (94i:14053)

Algebraic Structure and its Applications Research Center (ASARC), Department of Mathematics, Korea Advanced Institute of Science and Technology, 373-1 Gusungdong, Yusung-Gu, Daejeon, Republic of Korea

E-mail address: han.kangjin@kaist.ac.kr

Current address: School of Mathematics, Korean Institute for Advanced Study (KIAS), 85 Hoegiro, Dongdaemun-gu, Seoul 130-722, Republic of Korea

E-mail address: kangjin.han@kias.re.kr 